\title{
Acquisition of Cooperative Behavior in a Soccer Task Using Reward Shaping
}

\author{
Takashi Abe \\ Graduate School of Informatics and \\ Engineering, The University of \\ Electro-Communications, Tokyo, \\ Japan \\ abe.takashi@ohsuga.lab.uec.ac.jp
}

\author{
Ryohei Orihara \\ Graduate School of Informatics and \\ Engineering, The University of \\ Electro-Communications, Tokyo, \\ Japan \\ orihara@acm.org
}

\author{
Yuichi Sei \\ Graduate School of Informatics and \\ Engineering, The University of \\ Electro-Communications, Tokyo, \\ Japan \\ seiuny@uec.ac.jp
}

\author{
Yasuyuki Tahara \\ Graduate School of Informatics and \\ Engineering, The University of \\ Electro-Communications, Tokyo, \\ Japan \\ tahara@uec.ac.jp
}

\author{
Akihiko Ohsuga \\ Graduate School of Informatics and \\ Engineering, The University of \\ Electro-Communications, Tokyo, \\ Japan \\ ohsuga@uec.ac.jp
}

\begin{abstract}
In this research, soccer task is investigated among the numerous tasks of deep reinforcement learning. The soccer task requires cooperative behavior. However, it is difficult for the agents to acquire the behavior, because a reward is sparsely given. Moreover, the behaviors of the allies and opponents must be considered by the agents. In addition, in the soccer task, if the agents attempt to acquire high-level cooperative behavior from low-level movements, such as ball kicking, a huge amount of time will be needed to learn a model. In this research, we conduct experiments in which reward shaping is incorporated into deep reinforcement learning. This enables the agents to efficiently acquire cooperative behavior from low-level movements in a soccer task. The findings of this research indicate that reward shaping with a designer's domain knowledge positively influences the agent's attempt to acquire cooperative behavior from low-level movements.
\end{abstract}

\section{CCS CONCEPTS}

- Computing methodologies; • Machine learning; • Learning paradigms; • Reinforcement learning; • Multi-agent reinforcement learning;

\section{KEYWORDS}

Soccer, MuJoCo, Reward Shaping

\section{ACM Reference Format:}

Takashi Abe, Ryohei Orihara, Yuichi Sei, Yasuyuki Tahara, and Akihiko Ohsuga. 2021. Acquisition of Cooperative Behavior in a Soccer Task Using Reward Shaping. In 2021 the 5th International Conference on Innovation in

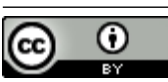

This work is licensed under a Creative Commons Attribution International 4.0 License.

ICIAI 2021, March 05-08, 2021, Xia men, China

(c) 2021 Copyright held by the owner/author(s).

ACM ISBN 978-1-4503-8863-4/21/03.

https://doi.org/10.1145/3461353.3461360
Artificial Intelligence (ICIAI 2021), March 05-08, 2021, Xia men, China. ACM, New York, NY, USA, 6 pages. https://doi.org/10.1145/3461353.3461360

\section{INTRODUCTION}

\subsection{Multi-agent Reinforcement Learning}

In recent years, deep reinforcement learning, which combines deep learning and reinforcement learning, has been widely used in game AI [1][2] and robot control [3][4]. However, its application in the real world still faces numerous challenges. In the real world, few tasks can be completed by a single agent, and cooperative behavior of multiple agents is required. Reinforcement learning that involves multiple agents is known as multi-agent reinforcement learning.

Multi-agent tasks are encountered in many real-world situations, such as peer-to-peer ridesharing system [5] and traffic signal control [6]. Li et al. addressed the order dispatching problem in the peer-topeer ridesharing problem [5]. In the paper, they adopted the mean field approximation to simplify the local interactions, and their proposed method performed better than several strong baselines in the accumulated driver income (ADI) and order response rate measures. Prabuchandran et al. applied multi-agent reinforcement learning algorithms to obtain dynamic traffic signal control policies, and modeled each traffic signal junction as an independent agent [6]. They showed through VISSIM that their algorithms performed better than the standard fixed signal timing (FST) algorithm and the saturation balancing (SAT) algorithm at two real road networks. As described, multi-agent reinforcement learning is being studied to utilize reinforcement learning in the real world.

\subsection{Soccer Task}

Among the tasks of multi-agent reinforcement learning, the soccer task is most frequently used in literatures. In this task, elucidating which actions are likely to bring a reward and a punishment is difficult, as agents can only receive the reward or the punishment when they score a goal or they lose a point. In tasks in which the agents have few opportunities to receive rewards, learning is difficult [7]. Furthermore, in the soccer task, the agents find it difficult to score goals and prevent goals by themselves; thus, a 
cooperative and hostile behavior is necessary. Unlike learning by a single agent, the agents must select the optimal action, taking into consideration the behavior of the allies and opponents. Therefore, learning is difficult as the amount of information that needs to be considered increases, and the exploration takes a huge amount of time.

To date, a number of researches on reinforcement learning of the soccer task have been conducted. In particular, the Keepaway task [8] in RoboCup Soccer [9] is incorporated by numerous researchers. In this task, the agents are divided into the keepers team and the takers team. The keepers team holds the ball, so that the takers team cannot steal the ball. In most researches on the soccer task, including the Keepaway task, agents learn to acquire a cooperative and hostile behavior through manually designed complex movements in advance. However, with regard to tasks with opponents, such as soccer simulations, human development efforts may be insufficient to outperform the opponent teams benefitting from the implementation by more knowledgeable designers [10]. This is because the designer's implementation ability is greatly reflected in the match results. Furthermore, if low-level movements, such as ball kicking and moving to the appropriate position, are designed in advance, the agents will be only able to acquire cooperative and hostile behavior based on the designer'sperspective, thus making it difficult to obtain knowledge beyond the human knowledge.

In this paper, we consider a learning method that enables agents to effectively acquire cooperative behavior in a soccer task, in which it is difficult for the agents to acquire cooperative behavior from low-level movements. We aimed to enhance learning efficiency using reward shaping [11] to overcome a problem, in which a huge amount of time is needed for the agents to acquire an appropriate behavior in the soccer task. In addition, our research enables agents to acquire cooperative behavior by combining reward shaping with the REINFORCE algorithm [12] in a soccer task implemented using a physics engine MufoCo [13] created by DeepMind.

\section{RELATED WORK}

So far, numerous researches on soccer task have been conducted, some of which enabled agents to acquire high-level behavior from low-level movements via reinforcement learning. Peng et al. [14] enabled agents to acquire dribbling skills by using hierarchical reinforcement learning, and Riedmiller et al. [10] conducted a research that allowed agents to acquire defensive behavior. However, when dribbling in an actual game, the agent has to carry the ball, taking into consideration the position of the allies and opponents. It is difficult for the agents to incorporate the dribbling skill learned in the environment without allies nor opponents into the cooperative behavior. In Riedmiller et al.'s research, in which agents acquired defensive behavior, there is a problem in the agent's ability to acquire cooperative behavior, taking into consideration the zone defense or the movements of the allies.

With the aim of solving these problems, Chitnis et al. investigated the agent's skills in passing the ball and receiving the pass from low-level movements [15]. They employed intrinsic motivation to encourage cooperative behavior. In this task, an episode ends when two agents are involved in a ball and then score a goal, or a certain amount of steps has passed. However, to acquire the cooperative behavior, it takes 100,000 episodes by using parallel learning of 30 agents. Furthermore, Liu et al. studied the reinforcement learning of the soccer task from low-level movements in a game format having two allies and two opponents [16]. In this research, the cooperative behavior of the agent is acquired using an algorithm combining SVG0 [17] and Population-Based Training [18]. In this task, an episode ends when either team scores a goal or 45 seconds have passed. However, to acquire pass skill or intercept skill, it takes 80 billion episodes in this learning. Therefore, since a huge amount of time is required to solve these problems, an appropriate approach for learning the task of acquiring cooperative behavior from low-level movements in a soccer task needs to be established.

\section{REINFORCEMENT LEARNING}

Reinforcement learning is a learning method in which an agent observes the environment and maximizes the rewards for the actions the agent has taken. The total rewards that can be obtained by the agent in the future are defined as the value of the agent's action. In addition, the agent learns the policy, so that the policy maximizes the value of the agent's action. When the agent learns the policy, the method for the calculation of the value and selection of the policy that maximizes the value is called value-based method. Conversely, the method for deciding and improving the policy to enhance its value is called policy-based method.

\subsection{Policy Gradient Method}

To optimize the agent's action, a method can be used to identify the optimal policy called policy iteration. Policy iteration is a method that identifies the optimal policy by repeating the policy evaluation step of calculating the value function under a policy as well as the policy improvement step of updating the policy to enable maximization of the value function [19].

In a model-free environment, the policy gradient method is employed as an approach different from policy iteration. It is a policy-based method that updates $\theta$ in the direction of the gradient $\nabla_{\theta} J(\theta)$ of the policy parameter $\theta$ in the objective function $J(\theta)$ and improves it, so that the objective function $J(\theta)$ takes a larger value. The gradient $\nabla_{\theta} J(\theta)$ is presented below [20]:

$$
\nabla_{\theta} J(\theta)=\mathbb{E}_{\pi}\left[\nabla_{\theta} \log \pi\left(A_{t} \mid S_{t}, \theta\right) Q_{\pi}\left(S_{t}, A_{t}\right)\right]
$$

Here, $Q_{\pi}\left(S_{t}, A_{t}\right)$ denotes the value function when the agent takes action $A_{t}$ in the state $S_{t}$ at time $t$. Through this method, it becomes possible to improve the policy by incorporating the policy evaluation into the $\mathrm{Q}$ function.

\subsection{REINFORCE}

REINFORCE [12] is an algorithm that learns the policy through the approximation of the action-value function with the sum of the discounted rewards $G_{t}$ in the policy gradient method. Suppose an agent takes action $A_{t}$ in the state $S_{t}$ at time $t, R_{t+k}$ denotes the reward obtained in the future, and $\gamma$ denotes the discount rate. The sum of the discounted rewards $G_{t}$ and the gradient $\nabla_{\theta} J(\theta)$ with the policy parameter $\theta$ are presented below:

$$
\mathrm{G}_{t}=\sum_{k=1}^{T-t} \gamma^{k-1} R_{t+k}
$$




$$
\nabla_{\theta} J(\theta) \approx \frac{1}{T} \sum_{t=0}^{T-1} \nabla_{\theta} \log \pi\left(A_{t} \mid S_{t}\right) G_{t}
$$

\section{REWARD SHAPING}

In tasks in which agents sparsely receive rewards, learning policy is difficult and very inefficient due to the difficulty in obtaining hints to achieve a final target during the agent's learning. For such tasks, reward shaping [11], in which agents gradually receive rewards, has been proposed. For example, consider the task of opening the lid of a plastic bottle. Simply twisting the lid will rotate the entire plastic bottle, and the lid cannot be opened. Learning would be relatively easy if the agent receives not only the extrinsic reward when the lid of the plastic bottle is opened but also the shaping reward when the plastic bottle is gripped with the opposite hand or the lid is twisted.

However, learning will be affected if reward shaping is improperly set [11]. For example, when opening a plastic bottle, if the agents receive shaping rewards when the lid is twisted to the direction opposite to that in which it should be twisted, the wrong guidance will hinder an efficient learning. Therefore, the designer who has the domain knowledge of the task needs to decide how to give the agents the rewards.

\section{APPROACH}

\subsection{Problem Setting}

This paper incorporates reward shaping into the REINFORCE algorithm to enable the agents to efficiently acquire cooperative behavior from low-level movements using $\mathrm{MuJoCo}$ Soccer [13] Two experiments in distinct problem settings have been conducted in this research, as presented in Figure 1, to compare how learning proceeds in each case, with one involving the player's interaction and another without it. We conducted the first experiment with Environment 1 as a simple task, in which one of the two agents kicks a ball into the goal. In Environment 1, we consider that the final target is achieved when the agents score a goal, regardless of the movement of the ally. Environment 2 is similar that in the research by Chitnis et al. [15]. In this environment, we consider that the final target is achieved when two agents touch the ball and then score a goal. Even when humans play soccer, there are some situations, in which the probability of scoring a goal increases by selecting a pass in a shot chance. In Environment 2, we assume a situation in which such multiple agents cooperate, and evaluate whether reward shaping promotes multiple agents to take cooperative behavior.

\subsection{Approach Overview}

5.2.1 Learning Algorithm. In this research, the model is trained using the algorithm proposed by Itoh et al. based on the REINFORCE algorithm [19]. In this model, the policy function was implemented as a Gaussian policy. Figure 2 presents the whole picture of the learning algorithm in this research.

After the agents observe a state, they predict the optimum action using a stochastic policy based on the Gaussian model. Then, the observed state and reward are saved as history when they take an action. If the end condition is not fulfilled, the step will be continued. In this research, the agents received shaping reward, a reward that

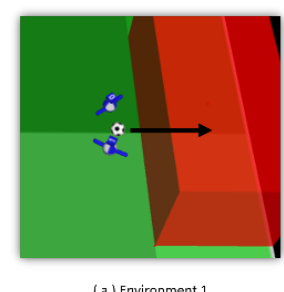

(a) Environment 1

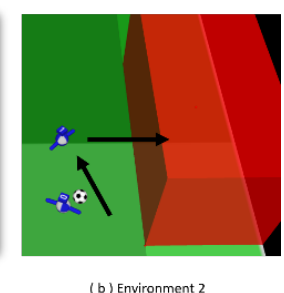

(b) Environment 2
Figure 1: Experimental environments

is given step by step until the final target is achieved, and extrinsic reward, which is a reward given when the final target is achieved. When an episode ends, the model is updated using states, actions, and rewards saved as a history for each step. To reduce the loss between the state value predicted by the agent based on the state at each step and the sum of the discounted rewards at each step, the state value function is updated. This function predicts the value of the state observed by the agent. In addition, the policy function is updated using the advantage function calculated based on the state value predicted by the state value function and the sum of the discounted reward. This function predicts the optimal action based on the state observed by the agent.

5.2.2 Reward Shaping. In this research, reward shaping can facilitate the agent in acquiring cooperative behavior in a soccer task. In Environment 2, the agent will fail to receive the reward for achieving the final target, even if the agent scores a goal by itself. Therefore, the cooperative behavior of the two agents is necessary. In this environment, reward shaping is employed to enable agents to efficiently acquire useful behavior in this environment.

However, as previously mentioned, due to the possibility that reward shaping negatively influences learning, how to give the agents rewards must be decided carefully. In this research, we give the agents extrinsic reward and shaping reward as follows, so that they can be actively involved in the ball.

Extrinsic Reward

When two agents are involved in the ball and then score a goal: $+10$

Shaping Reward

When the first agent is involved in the ball for the first time: +1

When the second agent is involved in the ball for the first time: $+1$

In this environment, due to the need for the agents to score a goal after the involvement of two agents in the ball, we need to make it easier for each agent to be involved in the ball. Thus, we give the agents rewards when each of them is involved in the ball. Here, the interaction of the two agents is encouraged by giving them rewards when the second agent is involved in the ball after another agent touched it, i.e., the second agent receives the pass. Furthermore, the shaping reward is set to be small and the extrinsic reward to be large to allow the agents to achieve the final target without being satisfied after they are involved in the ball. Moreover, we make it easier for the agents to achieve the final target. 


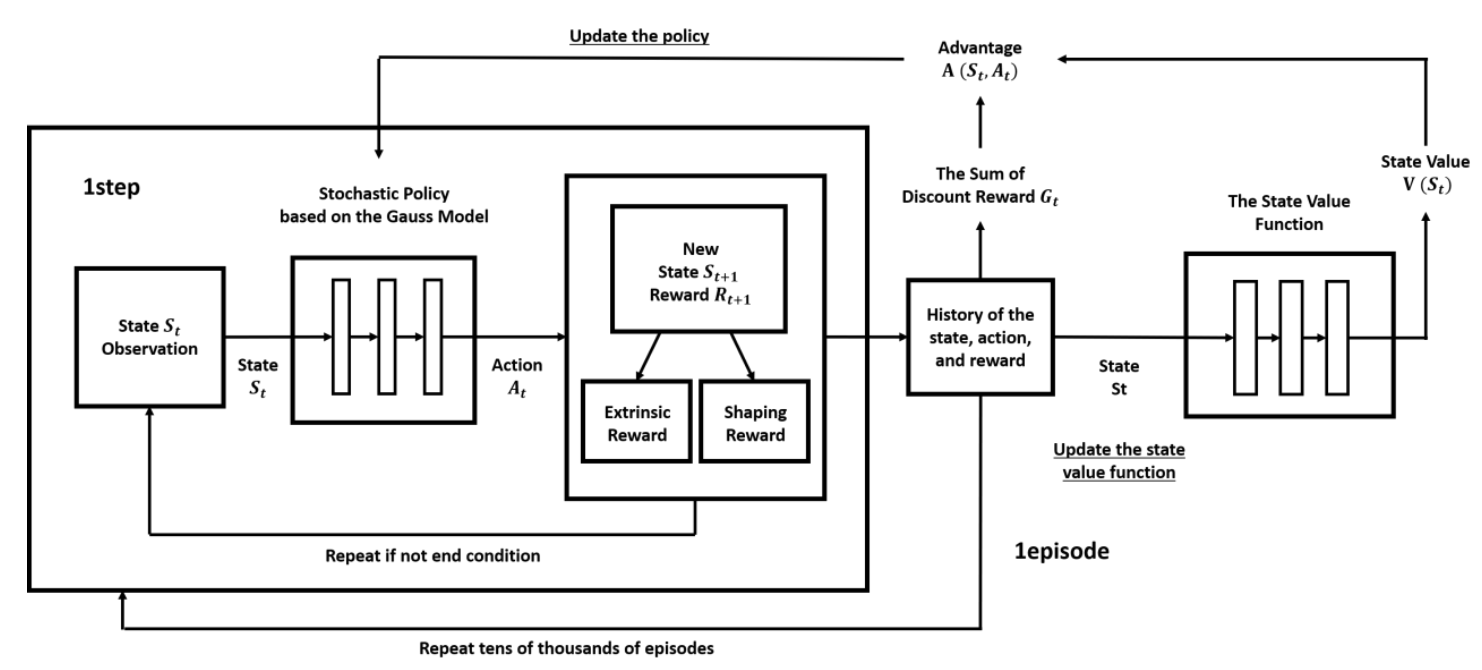

Figure 2: Learning algorithm in this research

\section{APPROACH}

\subsection{Experiment Summary}

In this research, a soccer task is simulated in $\mathrm{MuJoCo}$ Soccer, which is a physics engine developed by DeepMind. The soccer task is simulated under the following conditions [13].

- State: The state space has a dimension of 81 . The dimensions include the information of the proprioception, such as position, velocity, and accelerometer; the situation at a scene, such as an egocentric ball position, velocity and angular velocity, goal and corner positions; and teammate and opponent, such as orientation, position, and velocity.

- Action: The action space has a dimension of three. It includes accelerating the body forward-backward, rotating the body, and applying downward force to jump.

- Episode: One episode finishes when the final target is achieved or after 10s.

With regard to the policy, the input is the 81-dimensional state, and the output is the 3-dimensional action. The policy is implemented using a three-layer fully connected neural network with 810,220 , and 60-unit hidden layers, respectively. With regard to the value function model, the input is the 81-dimensional state, and the output is the 1-dimensional action value. The value function is implemented using a three-layer fully connected neural network with 810,63, and 5-unit hidden layers, respectively. The learning rate is 0.99 . RMSProp is employed in learning the policy as the optimization algorithm, and Adam is used in learning the value function model.

\subsection{Results}

6.2.1 Environment1. For Environment 1, in which the agents are unnecessary to interact with each other, learning efficiency is evaluated based on the average target achievement rates. The results are presented in Figure 3. In this task, the agents only receive the extrinsic reward when they achieve the final target, and learning is easily stabilized in an early stage. Even if the agents acquire a high-level behavior from low-level movements, it will be relatively easy for them to learn the high-level behavior without cooperative behavior. This is due to the relatively narrow state space which the agents consider comparing with Environment 2.

6.2.2 Environment2. For Environment 2, in which the agents need to interact with each other, learning efficiency is evaluated based on the average target achievement rates. Figure 4 presents the results from the two learning conditions, one with reward shaping and another without it. As a result of the learning, the average target achievement rate in the case with reward shaping is significantly higher than that without it.

In the early stage of learning up to 5000 episodes, target achievement rates with reward shaping rapidly increase from about 2000 episodes. Moreover, target achievement rates without reward shaping begins to increase for the first time in 3500 episodes. These experimental results indicate that reward shaping promotes the cooperative action of the two agents.

The experimental results without reward shaping indicate that acquiring the behavior from low-level movements is more difficult when agents need to interact than when they do not. Contrary to the case in which complex movements are implemented in advance, exploration is difficult due to many choices of the agent's actions when learning the behavior from low-level movements. In this environment, an agent who passes the ball must grasp the position of the ally and make a pass toward there; then, an agent who receives the ball must learn how to control the ball and score a goal. This series of actions need to be learned by two agents. Since the agents will find it difficult to learn if a reward is given only when all of these actions are completed, the use of reward shaping is suggested for this task to increase the number of times when the rewards are given.

Figure 5 presents the average of the total obtained reward, the average of the obtained shaping reward, and the average of the obtained extrinsic reward when using reward shaping in learning. Looking at the average of the obtained shaping reward, it can be 


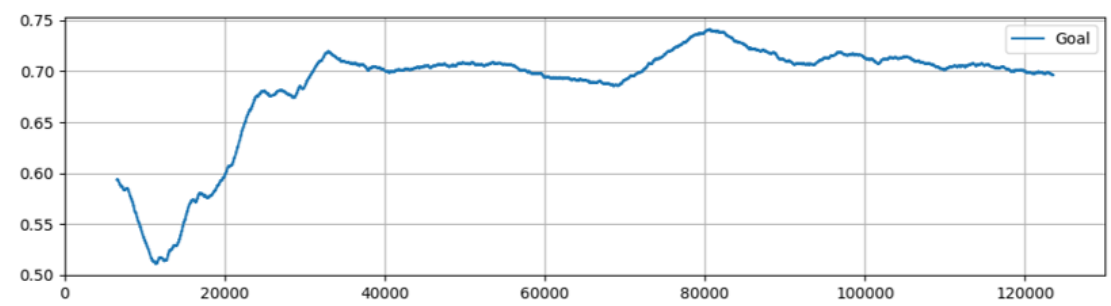

Figure 3: Average target achievement rates in Environment 1

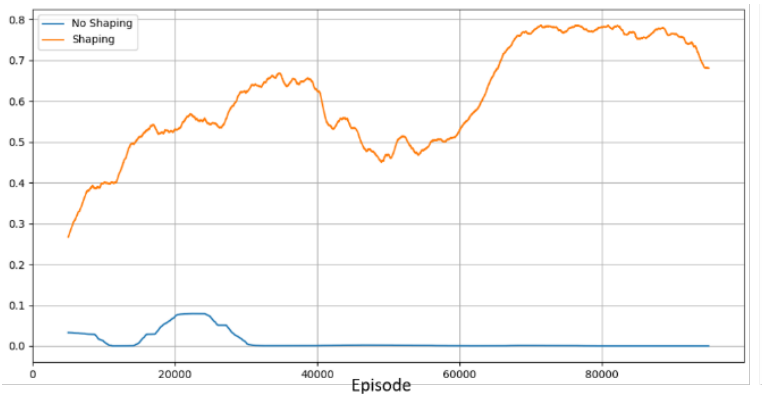

(a) Average target achievement rates until 100,000 episodes

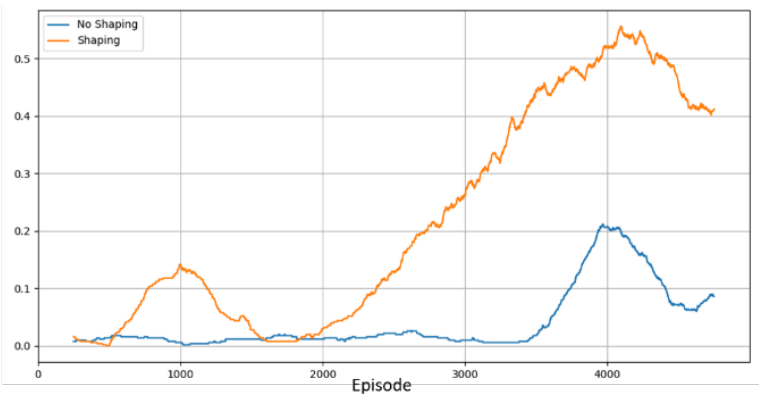

(b) Average target achievement rates until 5,000 episodes

Figure 4: Average target achievement rates in Environment 2

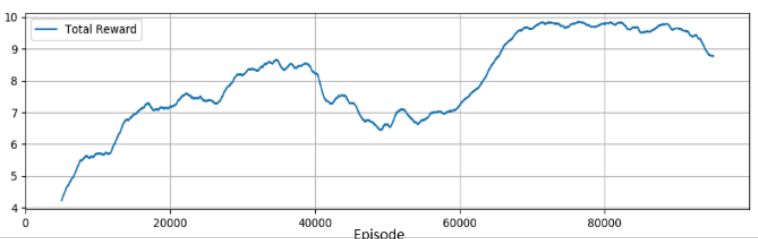

(a ) Average of the total obtained reward until 100,000 episodes

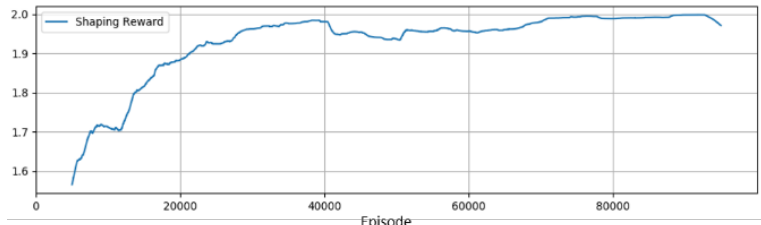

(c) Average of the obtained shaping reward until 100,000 episodes

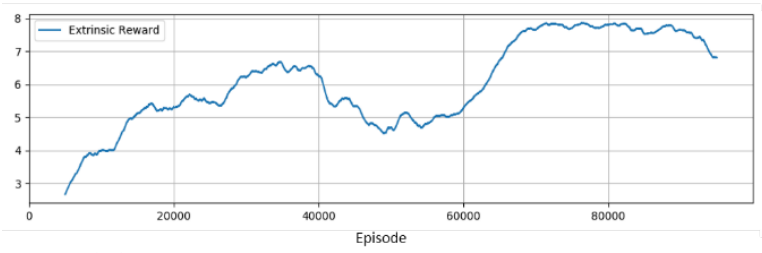

(e) Average of the obtained extrinsic reward until 100,000 episodes

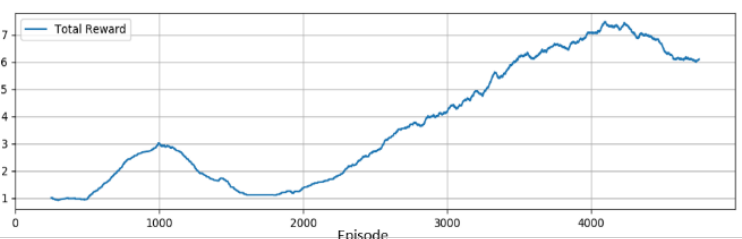

(b) Average of the total obtained reward until 5,000 episodes

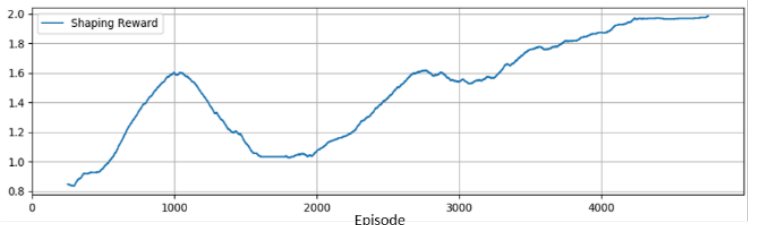

(d) Average of the obtained shaping reward until 5,000 episodes

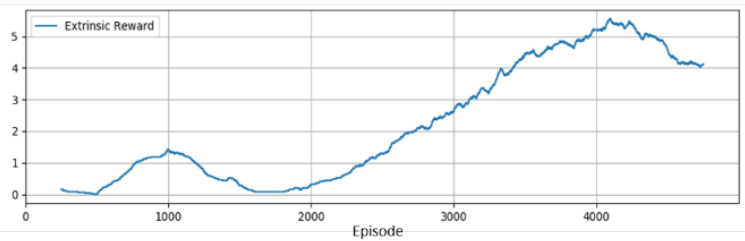

(f) Average of the obtained extrinsic reward until 5,000 episodes

Figure 5: Average of the obtained reward in the case of using reward shaping

determined that the reward finally converges to 2.0 , and the two agents seem to learn to be involved in the ball. In the early stage of learning, it is difficult for the agents to obtain 1.0 shaping reward. Moreover, there are episodes in which even one agent cannot be involved in the ball, but two agents can be involved in the ball after 5000 episodes. From this, it can be inferred that reward shaping positively influences learning in the early stage. 
Furthermore, the obtained shaping reward has increased without falling below 1.0 from 2,000 episodes. This resulted in the sharp increase of the obtained extrinsic reward from 2,000 episodes. From this observation, it can be inferred that if two agents are easily involved in the ball at the beginning, that is, the first agent finds it easier to kick the ball toward the second agent and increase the chances of the shoot, a higher target achievement rate can be obtained.

\section{CONCLUSION}

In this paper, a learning method that combines deep reinforcement learning with reward shaping in a soccer task, in which the agents must acquire cooperative behavior, is proposed. We conduct experiments using reward shaping to solve a problem that it takes agents a huge amount of time to acquire cooperative behavior. In this research, we show that the average target achievement rate in the case with reward shaping is significantly higher than that without it in soccer task, and confirmed that reward shaping can effectively facilitate the agent in acquiring cooperative behavior from low-level movements. In the future, how to promote learning efficiency by enabling agents to learn in different environments in stages will be studied.

\section{ACKNOWLEDGMENTS}

This work was supported by JSPS KAKENHI Grant Numbers JP17H04705, JP18H03229, JP18H03340, JP18K19835, JP19H04113, JP19K12107.

\section{REFERENCES}

[1] Volodymyr Mnih, Koray Kavukcuoglu, David Silver, Andrei A. Rusu, Joel Veness, Marc G. Bellemare, Alex Graves, Martin Riedmiller, Andreas K. Fidjeland, Georg Ostrovski, Stig Petersen, Charles Beattie, Amir Sadik, Ioannis Antonoglou, Helen King, Dharshan Kumaran, Daan Wierstra, Shane Legg, and Demis Hassabis. 2015 Human-level control through deep reinforcement learning. Nature 518, 7540 (February 2015), 529-533. https://doi.org/10.1038/nature14236

[2] David Silver, Aja Huang, Chris J. Maddison, Arthur Guez, Laurent Sifre, George van den Driessche, Julian Schrittwieser, Ioannis Antonoglou, Veda Panneershelvam, Marc Lanctot, Sander Dieleman, Dominik Grewe, John Nham, Nal Kalchbrenner, Ilya Sutskever, Timothy Lillicrap, Madeleine Leach, Koray Kavukcuoglu, Thore Graepel, and Demis Hassabis. 2016. Mastering the game of go with deep neural networks and tree search. Nature 529, 7587 (January 2016), 484-489. https://doi.org/10.1038/nature16961

[3] Timothy P. Lillicrap, Jonathan J. Hunt, Alexander Pritzel, Nicolas Heess, Tom Erez, Yuval Tassa, David Silver, and Daan Wierstra. 2015. Continuous control with deep reinforcement learning. 4th International Conference on Learning Representations (ICLR '15). 10 pages. http://arxiv.org/abs/1509.02971

[4] David Silver, Guy Lever, Nicolas Heess, Thomas Degris, Daan Wierstra, and Martin Riedmiller. 2014. Deterministic policy gradient algorithms. In Proceedings of the 31st International Conference on International Conference on Machine Learning - Volume 32 (ICML'14). 387-395. https://dl.acm.org/doi/10.5555/3044805 3044850
[5] Minne Li, Zhiwei Oin, Yan Jiao, Yaodong Yang, Jun Wang, Chenxi Wang, Guobin Wu, and Jieping Ye. 2019. Efficient Ridesharing Order Dispatching with Mean Field Multi-Agent Reinforcement Learning. In The World Wide Web Conference (WWW '19). Association for Computing Machinery, New York, NY, USA, 983-994. https://doi.org/10.1145/3308558.3313433

[6] Prabuchandran K.J., Hemanth Kumar A.N and S. Bhatnagar. 2014. Multi-agent reinforcement learning for traffic signal control. 17th International IEEE Conference on Intelligent Transportation Systems (ITSC '14). IEEE, 2529-2534. 10.1109/ITSC.2014.6958095

[7] Richard S. Sutton and Andrew G. Barto. 1998. Introduction to Reinforcement Learning. MIT Press, Cambridge, MA, USA

[8] Peter Stone, Richard S. Sutton, and Gregory Kuhlmann. 2005. Reinforcement learning for robocup soccer keepaway. Adaptive Behavior 13, 3 (September 2005), 165-188. http://nn.cs.utexas.edu/?AB05

[9] Hiroaki Kitano, Minoru Asada, Yasuo Kuniyoshi, Itsuki Noda, and Eiichi Osawa. 1997. Robocup: The robot world cup initiative. In Proceedings of the First International Conference on Autonomous Agents (AGENTS '97). Association for Computing Machinery, 340-347. https://doi.org/10.1145/267658.267738

[10] Martin Riedmiller, Thomas Gabel, Roland Hafner, and Sascha Lange. 2009. Reinforcement learning for robot soccer. Autonomous Robots 27, 1 (July 2009), 55-73. https://doi.org/10.1007/s10514-009-9120-4

[11] Andrew Y. Ng, Daishi Harada, and Stuart Russell. 1999. Policy invariance under reward transformations: Theory and application to reward shaping. Proceedings of the Sixteenth International Conference on Machine Learning (ICML '99). Morgan Kaufmann Publishers Inc., 278-287. https://dl.acm.org/doi/10.5555/645528.657613

[12] Ronald J. Williams. 1992. Simple statistical gradient-following algorithms for connectionist reinforcement learning. Mach. Learn. 8, 3-4 (May 1992), 229-256. https://doi.org/10.1007/BF00992696

[13] Emanuel Todorov, Tom Erez, and Yuval Tassa. 2012. Mujoco: A physics engine for model-based control. 2012 IEEE/RSJ International Conference on Intelligent Robots and Systems. IEEE, 5026-5033. https://doi.org/10.1109/IROS.2012.6386109

[14] Xue Bin Peng, Glen Berseth, KangKang Yin, and Michiel van de Panne. 2017. DeepLoco: Dynamic Locomotion Skills Using Hierarchical Deep Reinforcement Learning. ACM Trans. Graph. 36, 4, Article 41 (July 2017). https://doi.org/10.1145/ 3072959.3073602

[15] Rohan Chitnis, Shubham Tulsiani, Saurabh Gupta, and Abhinav Gupta. 2020. Intrinsic motivation for encouraging synergistic behavior. 8th International Conference on Learning Representations (ICLR '20). https://openreview.net/forum? id=SJleNCNtDH

[16] Siqi Liu, Guy Lever, Josh Merel, Saran Tunyasuvunakool, Nicolas Heess, and Thore Graepel. 2019. Emergent coordination through competition. 7th International Conference on Learning Representations (ICLR '19). http://arxiv.org/abs/ 1902.07151

[17] Nicolas Heess, Gregory Wayne, David Silver, Timothy Lillicrap, Tom Erez, Yuval Tassa. 2015. Learning continuous control policies by stochastic value gradients. In Proceedings of the 28th International Conference on Neural Information Processing Systems - Volume 2 (NIPS '15). MIT Press, Cambridge, MA, USA, 2944-2952. https://arxiv.org/abs/1510.09142

[18] Max Jaderberg, Valentin Dalibard, Simon Osindero, Wojciech M. Czarnecki, Jeff Donahue, Ali Razavi, Oriol Vinyals, Tim Green, Iain Dunning, Karen Simonyan, Chrisantha Fernando, and Koray Kavukcuoglu. 2017. Population based training of neural networks. http://arxiv.org/abs/1711.09846.

[19] Taichi Itoh, Yoshimitsu Imazu, Kodai Sudo, Masato Ninohira, Yusuke Kawasaki, Yuki Sakai, and Chungche Wei. You can use this in your field! Introduction to Deep Reinforcement Learning using Python, Exploration and Control by Reinforcement Learning. Shoeisha, 2019. (in Japanese)

[20] Richard S. Sutton, David McAllester, Satinder Singh, and Yishay Mansour. 1999. Policy gradient methods for reinforcement learning with function approximation. In Proceedings of the 12th International Conference on Neural Information Processing Systems (NIPS '99). MIT Press, Cambridge, MA, USA, 1057-1063. https://dl.acm.org/doi/10.5555/3009657.3009806 